\title{
A LOW COMPLEX MODIFIED GOLDEN CODE IN A COOPERATIVE RELAYING SYSTEM
}

\author{
K.Thilagam ${ }^{1}$ and K.Jayanthi ${ }^{2}$ \\ ${ }^{1}$ Research Scholar, Department of ECE, Pondicherry Engg. College, Puducherry, India \\ ${ }^{2}$ Associate Professor, Department of ECE, Pondicherry Engg. College, Puducherry,India
}

\begin{abstract}
In recent years, cooperative communication is a hot topic of research and it is a powerful physical layer technique to combat fading in wireless relaying scenario. Concerning with the physical layer issues, in this paper it is focussed on with providing a better space time block coding (STBC) scheme and incorporating it in the cooperative relaying nodes to upgrade the system performance. Recently, the golden codes have proven to exhibit a superior performance in a wireless MIMO (Multiple Input Multiple Output) scenario than any other code. However, a serious limitation associated with it is its increased decoding complexity. This paper attempts to resolve this challenge through suitable modification of golden code such that a less complex sphere decoder could be used without much compromising the error rates. The decoder complexity is analyzed through simulation and it proves to exhibit less complexity compared to the conventional (Maximum likelihood) ML decoder. The single relay cooperative STBC consisting of source, relay and destination are considered. The cooperative protocol strategy considered in the relay node is Decode and forward (DF) protocol. The proposed modified golden code with less complex sphere decoder is implemented in the nodes of the cooperative relaying system to achieve better performance in the system. The simulation results have validated the effectiveness of the proposed scheme by offering better BER performance, minimum outage probability and increased spectral efficiency compared to the non cooperative transmission method.
\end{abstract}

\section{KEYWORDS}

Cooperative communication, cooperative STBC, Bit error rate (BER), outage probability, spectral efficiency, Golden code, low complex sphere decoder.

\section{INTRODUCTION}

In wireless communication, there is an ever growing demand for higher data rate, highly reliable transmission schemes and increased spectral efficiency for high speed wireless connectivity. The multiple input multiple output (MIMO) technique is a promising scheme for reliable communication at higher data rate by offering spatial diversity and increase in capacity without the additional power consumption or bandwidth expansion. Cooperative communication is an interesting research topic in wireless communication because it achieves distributed spatial diversity, wider coverage, low transmit power and reduced interference. Recently, a new technique which exploits the advantages of aforementioned schemes through MIMO techniques and cooperation (i.e., combining space time block coding and cooperative relaying) attracts a widespread attention in the literature. The alamouti coded form constructed at the destination node and each relay node performs either time-reversal or conjugation operation (i.e.) the modified operations at the relay nodes which overcomes the influence of the frequency selective fading channels has been discussed in [1]. A ML decoder for the DF-based differential 
cooperative system utilizing complex-valued unitary and non-unitary constellations and uncoded transmissions. Also, deriving a (piecewise linear) PL decoder for complex-valued unitary, nonunitary constellations and closed-form expression of the approximate uncoded symbol error rate (SER) has been discussed in [2]. In [3] discussed about the signal designs such as space time codings / modulations to achieve full cooperative diversity and an overview of the efforts on combating the time and frequency asynchronism of the cooperative communication network. The joint source channel coding (JSCC) scheme for scalable video transmission over cooperative MIMO which was capable of dynamically adapting to the varying MIMO channel has been discussed in [4]. In [5] the authors discussed, about the application of adaptive modulation concept to the performance of cooperative techniques and to improve the spectral efficiency of the amplify and forward system. The measured bit error rate performance of a three node cooperative communication system operating with a maximum ratio combining technique and two cooperative coded schemes using hard and soft decision decoding has been discussed in [6]. The Distributed space time trellis codes (DSTTC) design criteria formulation with perfect and imperfect decoding at the relay and the derivation of the upper bounds of pair wise error probability (PEP) for different channel condition has been discussed in [7].

In [8], the authors has discussed the Double-differential (DD) modulation for amplify and forward (AAF) cooperative communications system that works well with carrier offsets and further the numerical power allocation improves the performance of the system. The Distributed source coding, channel coding and modulation(DSCM) designed for ensuring the decoding convergence of its constituent component codes, the variable length code(VLC) and two coded modulation (CM) schemes for cooperative communications has been discussed in [9]. An efficient space time coded cooperative relay communications scheme that employs linear precoding and a distributed relay selection algorithm to maximize the signal to noise ratio at the receiver has been discussed in [10].In [11], the space time block codes for a half-duplex multiple input multiple output (MIMO) and non-orthogonal amplify and forward (NAF) cooperative diversity scheme with multiple relays has been discussed. The achievable rates and fairness for several protocols using rateless codes has been discussed in [12]. In [13], the authors discussed about the relay using AF method and the source constructed with a distributed alamouti space time code and each terminal transmitted with the row of alamouti code. The design of space time codes that are capable of achieving the full diversity and by illustrating how cooperation among nodes with different numbers of antennas can be accomplished and how the quality of interuser link can be affected has been discussed in [14].

For a 2x2 MIMO system, golden code was discussed as a full rate and full diversity code with Non-vanishing minimum determinant (NVD) in [15].In [16], the implementation of the golden ratio encoder such that the exponential accuracy is robust against the threshold effects using Directed acyclic graphs (DAG) model has been discussed. A new ML decoding algorithm for the golden code which retains its fast decodable property on time varying channels and its comparison with the overlaid alamouti code has been discussed in [17]. In [18] the authors discussed, a method to develop the suboptimal decoders for the golden code with reduced complexity and near -ML performance, as the ML- decoding of the golden code have higher complexity. In [19], the design of low complex Maximum likelihood (ML) decoder for Space Time Block Codes (STBC) and embedded orthogonal STBC with symmetric and asymmetric coding conditions has been discussed. Multigroup maximum-likelihood (ML) decoding for collocated and distributed space-time block codes (DSTBCs) and the orthogonal frequencydivision multiplexing (OFDM)-based Alamouti space-time coded scheme application in synchronous wireless relay networks has been discussed in [20]. A suitable modification of golden code with less complex sphere decoder was proposed to achieve the better BER performance compared to the conventional space time block codes has been analysed and discussed in [21].In this paper, the proposed modified golden codes with less complex sphere decoder in [21] is implemented in the nodes of cooperative relaying system and its performance is 
analyzed. The simulation results shows that the proposed scheme under cooperative relaying scenario offers better BER performance, minimum outage probability and maximum spectral efficiency compared to the non-cooperative transmission scheme.

The remaining part of the paper is organized as follows: In section 2, the system model of the proposed cooperative relaying scheme is elaborated. In section 3, the proposed modified golden code is discussed analytically. Section 4, elaborates the decoder design for the proposed setup. Simulation results are discussed in Section 5. Section 6, deals with the conclusion of the paper.

\section{SYSTEM MODEL OF THE PROPOSED COOPERATIVE RELAYING SCHEME}

Consider a cooperative communication network consisting three nodes, the source(S) node, relay(R) node and destination(D) node each has two antennas. Let ' $h_{s, r}$ ' be the fading channel matrix between the $S$ and $R$, ' $h_{s, d}$ ' be the fading channel matrix between $S$ and $D$, and ' $h_{r, d}$ ' be the fading channel matrix between $\mathrm{R}$ and $\mathrm{D}$. Since the assumption is two antennas, all the fading channel matrices are $2 \times 2$ matrices whose entries are independent complex Gaussian random variables with zero mean and unit variance. The terms $h_{s, r}^{i, j}, h_{r, d}^{i, j}$ and $h_{s, d}^{i, j}$ for $i, j=1,2$ are the fading coefficients from the $i^{\text {th }}$ transmit antenna to the $j^{\text {th }}$ receive antenna for the corresponding channels. Further, the assumptions made are cellular mobile environment, the rayleigh fading for all channels and perfect channel state information is known at the receiver. It is also assumed to have half duplex communication that is, all nodes can either transmit or receive and two phase cooperative communications. Cooperative protocol strategies are classified by the operation at $\mathrm{R}$ and here it is assumed to be $\mathrm{DF}$ protocol, where $\mathrm{R}$ decodes the received signal, reencodes, and transmits to D.

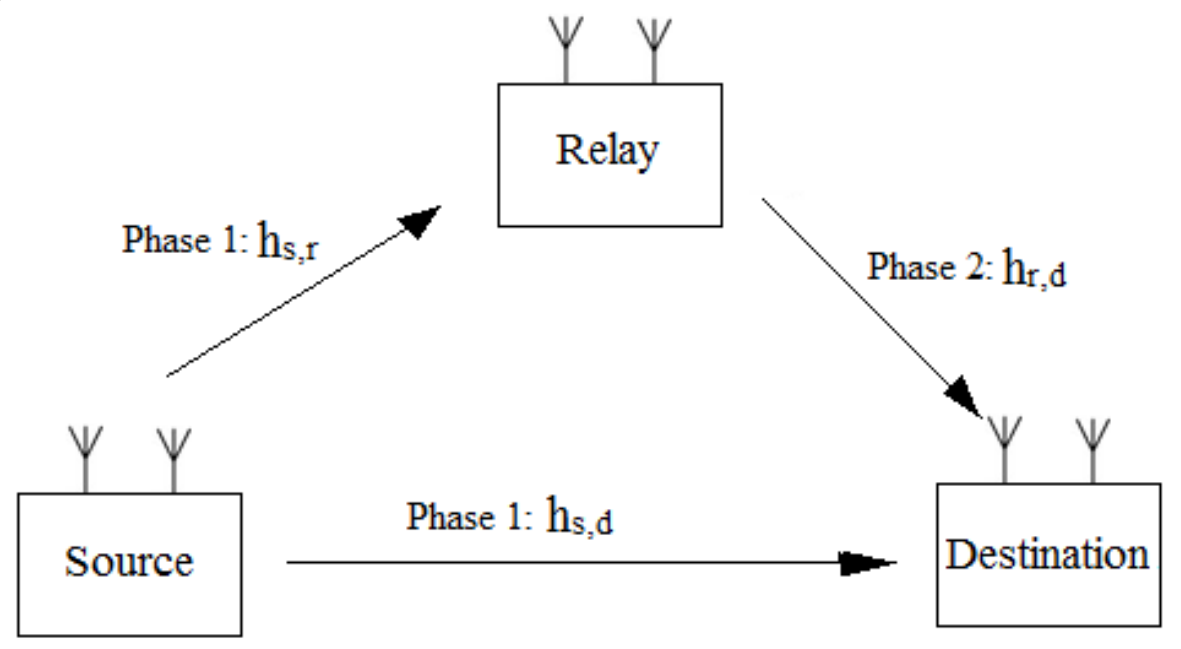

Figure 1. Cooperative Communication for single relay with the Implementation of Proposed low complex modified golden codes in the nodes

Referring to the Figure.1, the system model of the proposed scheme has the source node, relay node and the destination node each having two antennas. The cooperation strategy is modeled with two orthogonal phases to avoid the interferences. In phase 1, source sends information to the destination and the information is received by the relay at the same time. In phase 2, the relay helps the source by forwarding or retransmitting the information to the destination. In phase 1, the source broadcasts its information to both the destination and the relay. The received signals at the destination and the relay are obtained as, 
International Journal of Computer Networks \& Communications (IJCNC) Vol.6, No.1, January 2014

$$
\begin{aligned}
& y_{s, d}=\sqrt{P} h_{s, d} x+n_{s, d} \\
& y_{s, r}=\sqrt{P} h_{s, r} x+n_{s, r}
\end{aligned}
$$

In phase 2, the relay forwards a processed version of the source's signal to the destination and this is obtained as,

$$
y_{r, d}=h_{r, d} q\left(y_{s, r}\right)+n_{r, d}
$$

the function $\mathrm{q}($.$) depends on which processing is implemented at the relay node.$

Where, ' $\mathrm{P}$ ' is the total transmit power at the source, ' $\mathrm{x}$ ' is the transmitted information symbol, ' $n_{\mathrm{s}, \mathrm{d}}$ ', ' $\mathrm{n}_{\mathrm{s}, \mathrm{r}}$ ' and ' $\mathrm{n}_{\mathrm{r}, \mathrm{d}}$ ' are the additive noises, ' $\mathrm{h}_{\mathrm{s}, \mathrm{d}}$ ', ' $\mathrm{h}_{\mathrm{s}, \mathrm{r}}$ ' and ' $\mathrm{h}_{\mathrm{r}, \mathrm{d}}$ ' are the channel coefficient from source to destination, source to relay and relay to destination respectively. They are modelled as zero-mean, complex Gaussian random variables with variances $\boldsymbol{\sigma}_{s, d}^{2}, \quad \boldsymbol{\sigma}_{s, r}^{2}$ and $\boldsymbol{\sigma}_{r, d}^{2}$ respectively. With the variance ' $\mathrm{N}_{\mathrm{o}}$ ' the noise terms ' $\mathrm{n}_{\mathrm{s}, \mathrm{d}}$ ', ' $\mathrm{n}_{\mathrm{s}, \mathrm{r}}$ ' and ' $\mathrm{n}_{\mathrm{r}, \mathrm{d}}$ ' are modeled as zeromean, complex Gaussian random variables. The mutual information in terms of channel fades for decode and forward transmission is given by,

$$
I_{D F}=\frac{1}{2} \min \left\{\log \left(1+\Gamma\left|h_{s, r}\right|^{2}\right), \log \left(1+\Gamma\left|h_{s, d}\right|^{2}+\Gamma\left|h_{r, d}\right|^{2}\right)\right\}
$$

Considering rayleigh fading channel, the outage probability can be written as,

$$
\operatorname{Pr}\left[I_{D F}<R\right]=\operatorname{Pr}\left\{\left|h_{s, r}\right|^{2}<\frac{2^{2 R}-1}{\Gamma}\right\}+\operatorname{Pr}\left\{\left|h_{s, r}\right|^{2}>\frac{2^{2 R}-1}{\Gamma}\right\} \operatorname{Pr}\left\{\left|h_{s, d}\right|^{2}+\left|h_{r, d}\right|^{2}<\frac{2^{2 R}-1}{\Gamma}\right\}
$$

Averaging over the channel conditions, the outage probability for decode and forward is given by,

$$
\operatorname{Pr}\left[I_{D F}<R\right] \square \frac{1}{\sigma_{s, r}^{2}} \frac{2^{2 R}-1}{\Gamma}
$$

\subsection{Internal Structure of the Nodes}

This section elaborates the internal structures of the source node, relay node and destination node which are shown in Figure (a), Figure (b) and Figure (c).

Referring to the figure (a), the source node consists of two blocks namely QAM modulator block and RGR_GC encoder block. The operation at the source node is, the input bit streams are given as input to the QAM modulator and in the modulator bits are mapped according to the constellation and further encoded with RGR_GC encoder. Then, the encoded signal is launched over the two transmitting antennas. 
International Journal of Computer Networks \& Communications (IJCNC) Vol.6, No.1, January 2014

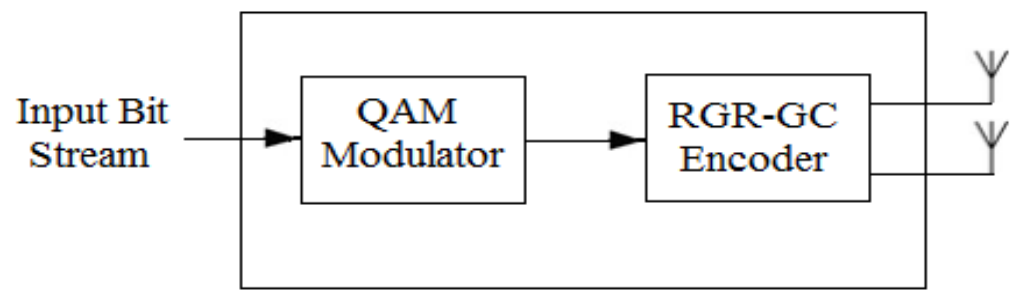

Figure (a). Source node

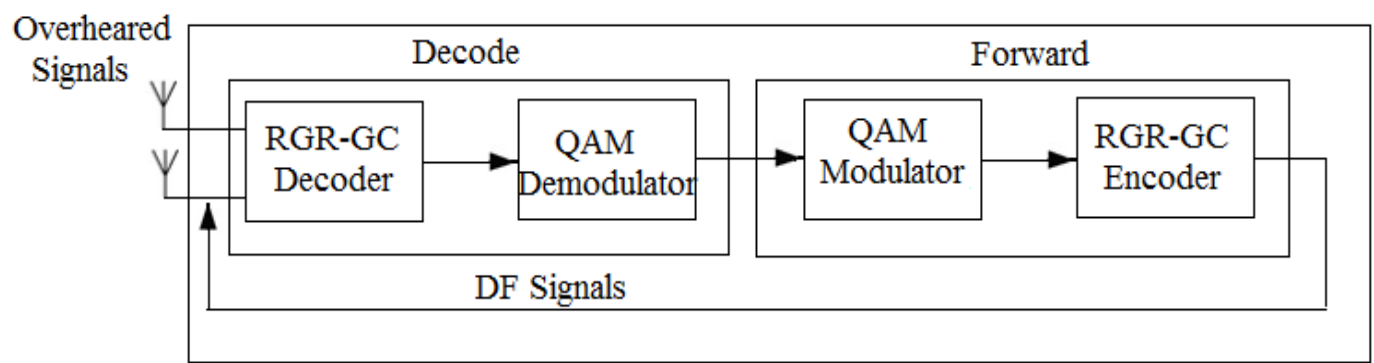

Figure (b). Relay node

Referring to the figure (b), the relay node consists of two blocks (i.e) the decoder block and forward block. The decoder block in turn has two blocks namely RGR_GC decoder and QAM demodulator. The forward block in turn has two blocks namely QAM modulator and RGR_GC encoder. The process involved is, the relay receives the overheard signals and it is given to the decoder block. In decoder block, the signal is decoded and demodulated. Then the decoder output is given as input to the forward block. The forward block re-encodes the input signal according to QAM constellation and generates the Decode and Forward (DF) signals. Further, the DF signal is launched over the two transmitting antennas.

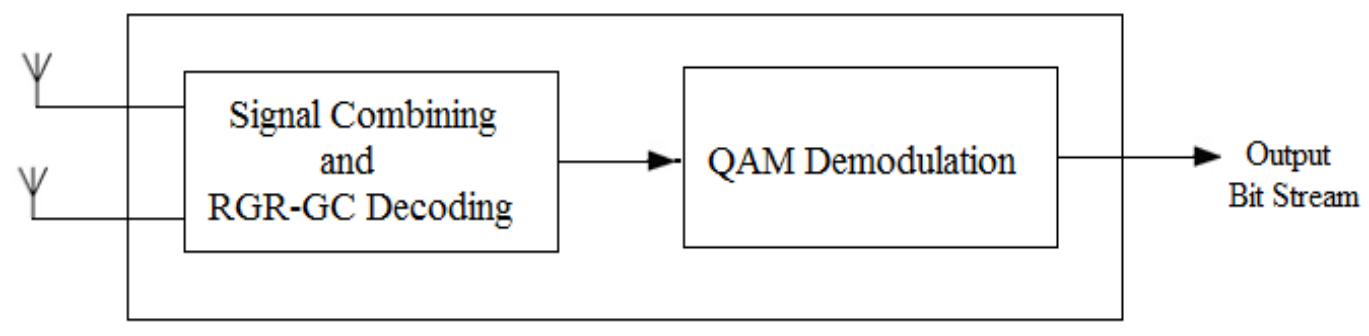

Figure (c). Destination node

Referring to the figure (c), the destination node consists of two blocks namely signal combining with RGR_GC decoding block and QAM demodulation block. The process involved is, the signals are received and combined using maximum ratio combiner and decoded using RGR_GC decoder. The decoder output is given as input to the QAM demodulator and finally the output bit streams are obtained. The next section deals with the proposed reduced golden ratio-golden code (RGR-GC) and its properties in detail. 


\section{Proposed Reduced Golden Ratio -Golden Code (RGR-GC)}

In this section, the proposed code and its properties satisfying golden codes are explained further. The RGR-GC is a scaled version of golden code which is used to normalize the energy and in turn, yields the performance improvement in the system. A reduced golden ratio-golden code (RGR-GC) is obtained by choosing a minimum polynomial equation in such a way that, it obtains the reduced golden ratio without losing golden codes key properties. The minimum polynomial equation considered to obtain the reduced golden ratio is

$\mathrm{X}^{2}-0.5 \mathrm{X}-0.5=0$.

$\theta=\frac{1+\sqrt{2}}{2}$, Where, ' $\theta$ ' is the reduced golden ratio

Having defined the polynomial and RGR, the next attempt is to focus onto shaping up the process to yield lossless property. A codeword from this algebra, before shaping is of the form,

$$
\left[\begin{array}{ll}
a+b \theta & c+d \theta \\
i(c+d \sigma(\theta)) & a+b \sigma(\theta)
\end{array}\right]
$$

where, the information symbols a,b,c,d $€ Z[i]$. The proposed code with Cyclic division Algebra (CDA) and its properties fulfilment are explained further.

Property 1: Cubic Shaping. This section adds cubic shaping to the codebook which provides the information lossless property. For Z[i]-lattice, the codeword is generated by the matrix,

$$
\left(\begin{array}{cc}
1 & \theta \\
1 & \sigma(\theta)
\end{array}\right)
$$

There will be some shaping loss as the matrix is not unitary. These problems can be fixed without losing the other algebraic properties. This issue can be addressed by lattice A with a 'M'generator matrix is given by,

$$
M=\left(\begin{array}{ll}
\alpha & 0 \\
0 & \sigma(\alpha)
\end{array}\right)\left(\begin{array}{cc}
1 & \theta \\
1 & \sigma(\theta)
\end{array}\right)=\left(\begin{array}{ll}
\alpha & \alpha \theta \\
\sigma(\alpha) & \sigma(\alpha) \sigma(\theta)
\end{array}\right)
$$

To make ' $\mathrm{M}$ ' as unitary matrix, the selection is made in such a way that $\alpha \in \mathrm{O}_{L}$

The lattice $\Lambda$ in the determinant form can be written as,

$$
\begin{aligned}
& \operatorname{det}(\Lambda)=\left|N_{L / K}(\alpha)\right|^{2}\left|d_{Q(\sqrt{2})}\right| \\
& =2\left|N_{L / K}(\alpha)\right|^{2}
\end{aligned}
$$

To have the lattice $Z[i]^{2}$, important condition is that the $\operatorname{det}(\Lambda)$ should be a square integer. To find such an element, the factorization of 2 in $\mathrm{O}_{\mathrm{L}}$ is given by,

$$
2=(1+i-i \theta)^{2}(1-i+i \theta)^{2}
$$


Choosing $(\alpha=1+i-i \theta)$.It can be checked whether the obtained is right lattice using the generator matrix $\mathrm{M}$, where $M^{\dagger}=2 I_{2}$ is obtained by direct computation. The unitary matrix is $\frac{1}{\sqrt{2}} M$ which yields the shaping property in the first layer and cubic shaping on the other layer. Adding the shaping property, the codeword ' $\mathrm{X}$ ' belonging to the RGR-GC has the form,

$$
X=\frac{1}{\sqrt{2}}\left[\begin{array}{ll}
\alpha(a+b \theta) & \alpha(c+d \theta) \\
i \sigma(\alpha)(c+d \sigma(\theta)) & \sigma(\alpha)(a+b \sigma(\theta))
\end{array}\right]
$$

Where a, b, c, d are QAM symbols and it takes any value in Z[i]. In an infinite code $\mathrm{C}_{\infty}, \mathrm{a}, \mathrm{b}, \mathrm{c}, \mathrm{d}$ can take any value in $\mathrm{Z}[\mathrm{i}]$ that is the finite signal constellations are carved from infinite lattices.

Property 2: Minimum Determinant. The computation of the minimum determinant of an infinite code is discussed next. Therefore,

$$
\begin{gathered}
X=\frac{1}{\sqrt{2}}\left[\begin{array}{ll}
\alpha & 0 \\
0 & \sigma(\alpha)
\end{array}\right]\left[\begin{array}{lr}
(a+b \theta) & (c+d \theta) \\
i(c+d \sigma(\theta)) & (a+b \sigma(\theta))
\end{array}\right] \\
\text { Where, } \alpha \sigma(\alpha)=1+i \\
\operatorname{det}(X)=\frac{1+i}{2}[(a+b \theta)(a+b \sigma(\theta))-i(c+d \theta)(c+d \sigma(\theta))] \\
=\frac{1}{1+i}\left[\left(a^{2}+a b-b^{2}-i\left(c^{2}+c d-d^{2}\right)\right)\right]
\end{gathered}
$$

where, $\left|a^{2}+a b-b^{2}-i\left(c^{2}+c d-d^{2}\right)\right|^{2}$ is 1 . As it is required by the NVD property, the minimum determinant of infinite code is bounded away from zero. To be noted is factor ' $i$ 'ensures that the average transmitted energy is uniform from both antennas in both channel uses and $|i|^{2}=1$,

Thus, $\quad \delta_{\text {min }}\left(C_{\infty}\right)=\min _{X \neq 0}|\operatorname{det}(X)|^{2}=\frac{1}{2}$

The next section explains a new low complexity decoder for the proposed code which is the major task of the golden codes.

\section{Proposed Low CoMplexity Sphere Decoder}

In this section, a new low complexity decoder for the Reduced Golden Ratio- golden codes (RGR-GC) is described. To reduce the decoding complexity, many methods have been analyzed. In [19], the low complexity decoding for the space time block codes and the key properties for reducing the decoding complexity was discussed in broad sense. One of the properties considered in the proposed method is, the real and imaginary parts of the symbol are decoded separately. Moreover, the channel matrix $\mathrm{H}=\mathrm{QR}$, was decomposed using $\mathrm{QR}$ decomposition method, where, ' $Q$ ' is a unitary matrix and ' $R$ ' is an upper triangular matrix. The ' $R$ ' matrix with zero entries and the real elements in it potentially reduce the decoding complexity. In the proposed method, the real and imaginary parts of the symbol are decoded separately using sphere decoder with tree search algorithm, in a single symbol manner for Reduced Golden ratio Golden codes (RGR_GC). The steps involved in the receiver side and algorithm used is explained further. At the receiver, 
International Journal of Computer Networks \& Communications (IJCNC) Vol.6, No.1, January 2014

the received vector of samples $y=\left[y_{1}[1], y_{2}[2], y_{3}[3], y_{4}[4]\right]^{T}$ with two antennas at two time instances can be obtained as,

$$
\mathrm{y}=\mathrm{Hx}+\mathrm{n}
$$

where, ' $\mathrm{x}$ ' is an transmitted symbol vector, ' $\mathrm{n}$ ' is the noise vector and ' $\mathrm{H}$ ' is the effective channel matrix represented as, $X=\left[x_{1}, x_{2}, x_{3}, x_{4}\right]^{T}, n=\left[n_{1}[1], \ldots . n_{2}[2]\right]^{T}$ and

$H=\left[\begin{array}{llll}h_{11}[1] & 0 & \phi h_{21}[1] & 0 \\ 0 & h_{21}[2] & 0 & \phi h_{11}[2] \\ h_{12}[1] & 0 & \phi h_{22}[1] & 0 \\ 0 & \phi h_{22}[2] & 0 & \phi h_{12}[2]\end{array}\right]\left[\begin{array}{llll}\alpha & \beta & 0 & 0 \\ -\beta & \alpha & 0 & 0 \\ 0 & 0 & \alpha & \beta \\ 0 & 0 & -\beta & \alpha\end{array}\right]$

respectively. where, $\phi=e^{j \frac{\pi}{4}}, \alpha=\cos (\theta), \beta=\sin (\theta)$

In the above equation, $\mathrm{H}=\mathrm{QR}$ is an orthogonal-triangular decomposition of the effective channel matrix where, $\mathrm{Q}$ is unitary and $\mathrm{R}$ is upper triangular. The Gram-Schmidt orthonormalization is followed for the decomposition. Where this procedure is applied to the columns of $\mathrm{H}$, then the entry of $R$ in row $i$ and column $j$ will be the inner product between the $i^{\text {th }}$ column of $Q$ and $j^{\text {th }}$ column of $H$. Therefore, $r_{i, j}=q_{i} * h_{j}$. In direct computation of $R$, the elements $r_{1,2}$ and $r_{3,4}$ are real, through the diagonal elements and the other elements are complex by nature. From the effective channel matrix, the diagonal elements $\left\{r_{1,1} \ldots . . r_{4,4}\right\}$ and nondiagonal elements $r_{1,2}$ and $r_{3,4}$ by direct computation is obtained as,

$$
\begin{aligned}
& r_{11}=\sqrt{\alpha^{2} \gamma_{1}+\beta^{2} \gamma_{2}} \quad, r_{12}=\frac{1}{r_{11} \sqrt{2}}\left(\gamma_{1}-\gamma_{2}\right), r_{22}=\frac{1}{r_{11}} \sqrt{\gamma_{1} \gamma_{2}} \\
& r_{33}=\sqrt{\frac{\alpha^{2}\left(\gamma_{3}+\gamma_{4}\right)}{\gamma_{1}^{2}}+\frac{\beta^{2}\left(\gamma_{5}+\gamma_{6}\right)}{\gamma_{2}^{2}}} \quad, \quad r_{34}=\frac{1}{r_{33} \sqrt{2}} \frac{\left(\gamma_{3}+\gamma_{4}\right)}{\gamma_{1}^{2}}-\frac{\left(\gamma_{5}+\gamma_{6}\right)}{\gamma_{2}{ }^{2}} \\
& r_{44}=\left(\frac{1}{r_{33} \gamma_{1} \gamma_{2}}\right)^{2} \sqrt{\left(\gamma_{3}+\gamma_{4}\right)\left(\gamma_{5}+\gamma_{6}\right)\left(\alpha^{2} \gamma_{2}{ }^{2}\left(\gamma_{3}+\gamma_{4}\right)+\beta^{2} \gamma_{1}^{2}\left(\gamma_{5}+\gamma_{6}\right)\right)}
\end{aligned}
$$

and so on.Decomposing the ' $\mathrm{R}$ ' and ' $\mathrm{X}$ ' matrix, it is got as,

$$
R=\left[\begin{array}{llll}
r_{11} & r_{12} & r_{13} & r_{14} \\
0 & r_{22} & r_{23} & r_{24} \\
0 & 0 & r_{33} & r_{34} \\
0 & 0 & 0 & r_{44}
\end{array}\right], \quad X=\left[\begin{array}{c}
x_{1}^{R}+j x_{1}^{I} \\
x_{2}^{R}+j x_{2}^{I} \\
x_{3}^{R}+j x_{3}^{I} \\
x_{4}^{R}+j x_{4}^{I}
\end{array}\right]
$$

The ' $\mathrm{R}$ ' matrix entries $\mathrm{r}_{11}, \mathrm{r}_{12}, \mathrm{r}_{22}, \mathrm{r}_{33}, \mathrm{r}_{34}, \mathrm{r}_{44}$ are real and in terms of $\mathrm{z}=\mathrm{Q} * \mathrm{y}$, the decoder chooses $\mathrm{x}$ to minimize,

$$
\begin{gathered}
P(x)=\|y-H x\|^{2} \\
=\|z-R x\|^{2}
\end{gathered}
$$

where, $\mathrm{P}(\mathrm{x})$ is the total cost metric.

$$
=\left\|z-R_{1} x_{1}-R_{2} x_{2}-R_{3} x_{3}-R_{4} x_{4}\right\|^{2}
$$

The decoder proceeds as follows and the total cost of (18) can be written as,

$$
P(x)=\left\|v-R_{1} x_{1}\right\|^{2}
$$


International Journal of Computer Networks \& Communications (IJCNC) Vol.6, No.1, January 2014

$$
\begin{aligned}
& =\left\|v^{R}-R_{1} x_{1}^{R}\right\|^{2}+\left\|v^{I}-R_{1} x_{1}^{I}\right\|^{2} \\
v & =z-R_{2} x_{2}-R_{3} x_{3}-R_{4} x_{4}
\end{aligned}
$$

The importance of going from equations (18) to (19) is the fact that $R_{1}$ is real. Therefore, for every candidate $\left(x_{1}, x_{2}, x_{3}, x_{4}\right)$ the fact that $\mathrm{R}_{1}$ is real and allows the receiver to decide on real

symbol $\left(x_{1}^{R}\right)$ from the imaginary symbol $\left(x_{1}^{\prime}\right)$ separately by minimizing $\left|v_{1}^{R}-r_{1,1} x_{1}^{R}\right|^{2}+\left|v_{1}^{I}-r_{1,1} x_{1}^{I}\right|^{2}$. The decoded symbol vector ' $\mathrm{x}$ ' will be the minimum of the overall cost metric $\mathrm{P}(\mathrm{x})$. The minimum of overall cost metric $\mathrm{P}(\mathrm{x})$ is obtained using the tree search algorithm. A sphere decoder with four level tree, for the reduced golden ratio-golden codes (RGR-GC) associated with different $\mathrm{x}_{\mathrm{i}}(\mathrm{i}=1, . .4)$ at each level has been discussed. It is proposed with a four level tree that associates $d^{R}=x_{4}{ }^{R}$ and $d^{I}=x_{4}{ }^{I}$ in the first level, $c^{R}=x_{3}{ }^{R}$ and $c^{I}=x_{3}{ }^{I}$ in the second level, $b^{R}=x_{2}{ }^{R}$ and $b^{I}=x_{2}{ }^{I}$ in the third level and $a^{R}=x_{1}{ }^{R}$ and $a^{I}=x_{1}{ }^{I}$ in the fourth level. Rewriting the cost metric function as,

$$
\begin{aligned}
& P(X)=\left\|V^{i}-A a^{I}\right\|^{2}+\left\|V^{i}-A b^{I}\right\|^{2}+\left\|V^{R}-A a^{R}\right\|^{2}+\left\|V^{R}-A b^{R}\right\|^{2} \\
& +\left\|Z_{{ }^{l}}{ }^{\prime}-D c^{l}\right\|^{2}+\left\|Z_{4}{ }^{l}-D d^{l}\right\|^{2}+\left\|Z_{{ }{ }^{R}}-D c^{R}\right\|^{2}+\left\|Z_{4^{R}}{ }^{R}-D d^{R}\right\|^{2}
\end{aligned}
$$

The number of combinations of each symbol $\left(\mathrm{x}_{1}\right)\left(\mathrm{x}_{2}\right)\left(\mathrm{x}_{3}\right)\left(\mathrm{x}_{4}\right)$ is denoted by $q$. The decoding complexity of $\sqrt{q}$, comes from the fact that the symbols $\left(\mathrm{x}_{1}{ }^{\mathrm{R}}\right)$ and $\left(\mathrm{x}_{1}{ }^{\mathrm{I}}\right)$ are separately decodable. Therefore, it has the decoding complexity of $\mathrm{O}\left(q^{1.5}\right)$. The next section deals with the tree search algorithm.

\subsection{Tree Search Algorithm}

The figure 2, represents the tree diagram of the proposed sphere decoder. As discussed before, the initial level of tree corresponds to bottom or the starting point of the tree called root node. Each node acts as parent node and the emerging node from the parent node is child node. The final stage of the tree structure is called leaf node. The total cost of a leaf node $\mathrm{x}$ decomposes into the sum of four branch metrics denoted by $\sum P_{i}$. Where, $P_{\mathrm{i}}$ represents the branch metric for a branch at the (4-i) ${ }^{\text {th }}$ stage of the tree. A sphere decoding algorithm for four level tree structures is briefed. The first few steps involves the initialization (i.e) QR decomposition of effective channel matrix, computation of ' $\mathrm{z}$ ' and the sphere radius $\hat{P}$ to infinity. It requires only two sorting operations and incorporates two common optimizations: updating the radius and pruning. The elements $\mathrm{k}, 1, \mathrm{~m}, \mathrm{n} € \Pi$ are the permuted channel matrix elements. In the first level of the decoder, the branch metric of the $\mathrm{P}_{4}$ is updated and in turn decides the $\mathrm{x}_{4}{ }^{\mathrm{R}}$ and $\mathrm{x}_{4}{ }^{\mathrm{I}}$. In the second level of the decoder, the branch metric of $\mathrm{P}_{3}$ is updated and $\mathrm{x}_{3}{ }^{\mathrm{R}}, \mathrm{x}_{3}{ }^{\mathrm{I}}$ are estimated. The intermediate values $\mathrm{V}_{2}, \mathrm{~V}_{1}$ are eliminated thereby reducing the interference. In the third level and the fourth level the branch metric $P_{2}$ and $P_{1}$ is updated with lowest value and estimates the $x_{2}{ }^{R}, x_{2}{ }^{I}$ and $x_{1}{ }^{R}, x_{1}{ }^{I}$ respectively. The overall cost for the current symbol vector is updated. If the current cost $\mathrm{P}$ is less than the previous smallest cost $\mathrm{P}$ then the radius and best candidate vector are updated, otherwise the process is terminated. This procedure is repeated cyclically to decode the symbols. 
International Journal of Computer Networks \& Communications (IJCNC) Vol.6, No.1, January 2014

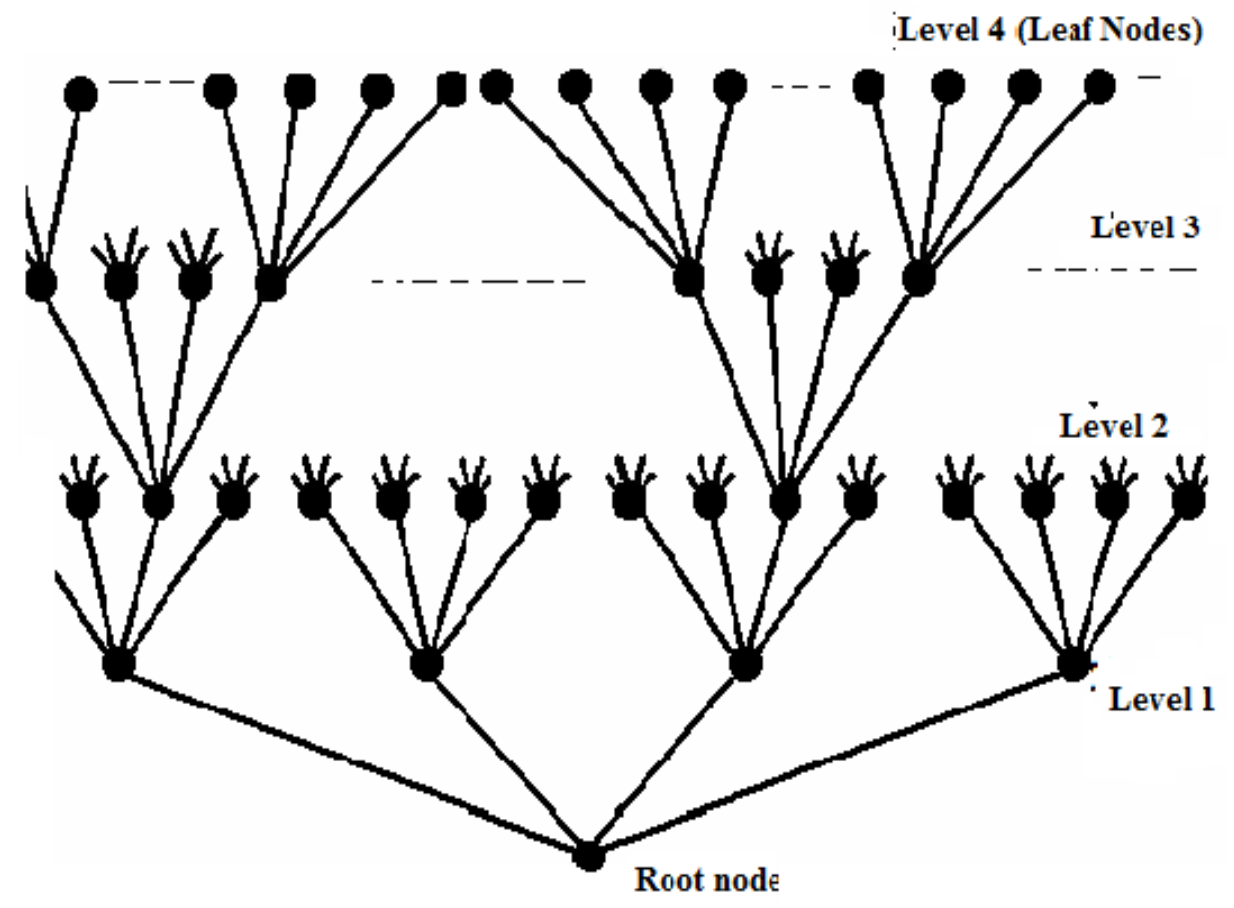

Figure 2. Tree representation of Proposed Sphere decoder

\section{Simulation ANALYSIS}

The simulation analysis for the proposed method is performed with the following parameters and it is tested in MATLAB 7.10 software. This section discusses the simulation analysis of the proposed scheme using the parameters mentioned in Table 1 . The complexity analysis of the proposed scheme is tested initially and further the performance analysis of the proposed scheme in cooperative relaying scenario is analyzed.

Table 1. Simulation parameters

\begin{tabular}{|c|c|}
\hline PARAMETERS & VALUES / TYPES \\
\hline Number of bits & $2 \times 10^{4}$ \\
\hline Number of Transmit antennas & 2 \\
\hline Number of Receive antennas & 2 \\
\hline Modulation schemes & 4-QAM,QPSK \\
\hline Code rate & $1,1 / 2$ \\
\hline Decoder Type & ML, RGR-GC sphere \\
\hline Coding Types & Alamouti, Golden, silver, RGR-GC \\
\hline
\end{tabular}


International Journal of Computer Networks \& Communications (IJCNC) Vol.6, No.1, January 2014

\begin{tabular}{|c|c|}
\hline Number of Transmitter & 1 \\
\hline Number of receiver & 1 \\
\hline Number of relay & 1 \\
\hline Power allocation & $\operatorname{Ps}=\operatorname{Pr}=(1 / 2) \mathrm{P}=0.5$ \\
\hline Relay location & Dsr=Dsd $=(1 / 2) \mathrm{Dsd}=0.5$ \\
\hline Path loss exponent & 2,3 \\
\hline Doppler frequency & 0.01 \\
\hline Channel Type & Rayleigh fading \\
\hline
\end{tabular}

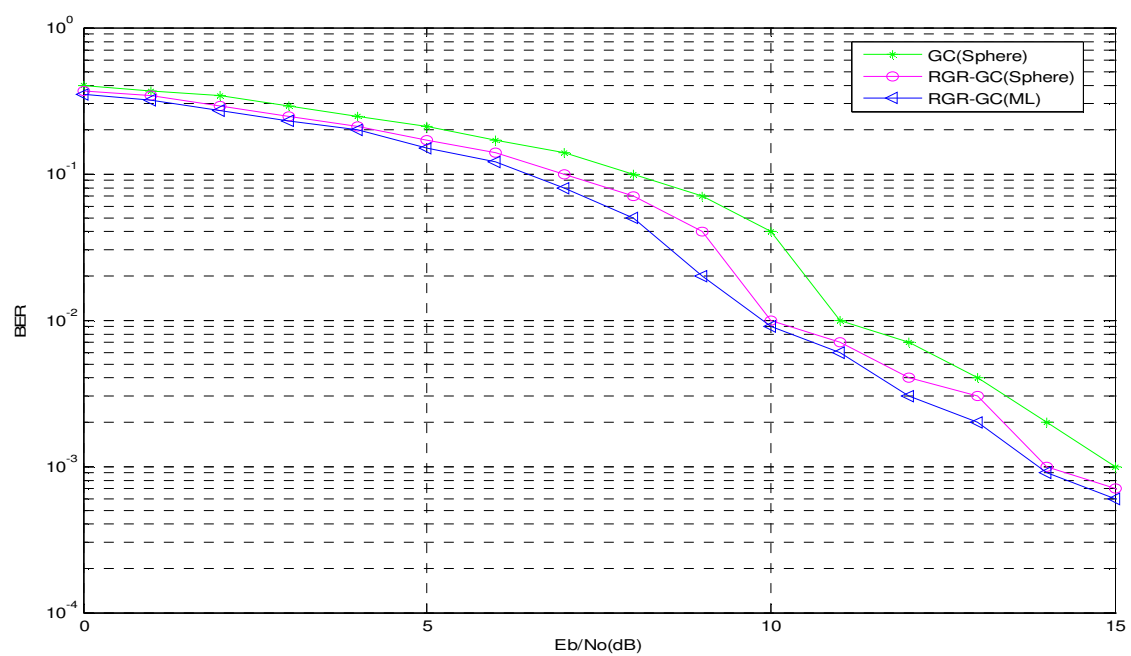

Figure 3. Performance comparison of Proposed (RGR-GC) sphere decoder with the ML decoder

From figure 3, the performance graphs were plotted for golden codes with proposed low complexity sphere decoder and proposed RGR-GC with conventional ML decoder. It is observed that the proposed RGR-GC with ML decoder achieves better BER performance compared to the other methods. In general ML decoder has better BER performance with less complexity for higher order modulations. So, it is claimed that the proposed RGR-GC sphere decoder achieves near ML performance with less computational complexity. 
International Journal of Computer Networks \& Communications (IJCNC) Vol.6, No.1, January 2014

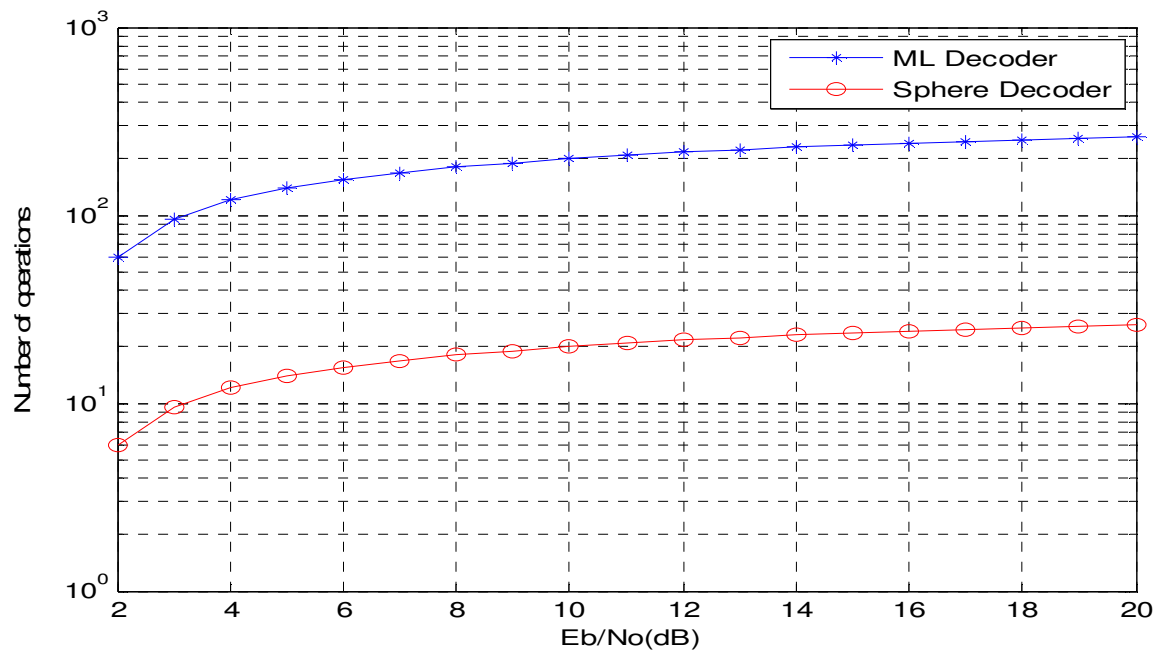

Figure 4. Complexity analysis for the proposed low complex sphere decoder and conventional ML decoder

The complexity analysis graphs were plotted for the proposed low complex sphere decoder and conventional ML decoder. From figure. 4, it is inferred from the graph that , for a fixed Eb/No of $3 \mathrm{~dB}$ the number of operations performed by the proposed low complex sphere decoder and conventional ML decoder is 10 (approx)and 100 (approx). The proposed scheme has less computational complexity compared to the conventional scheme.

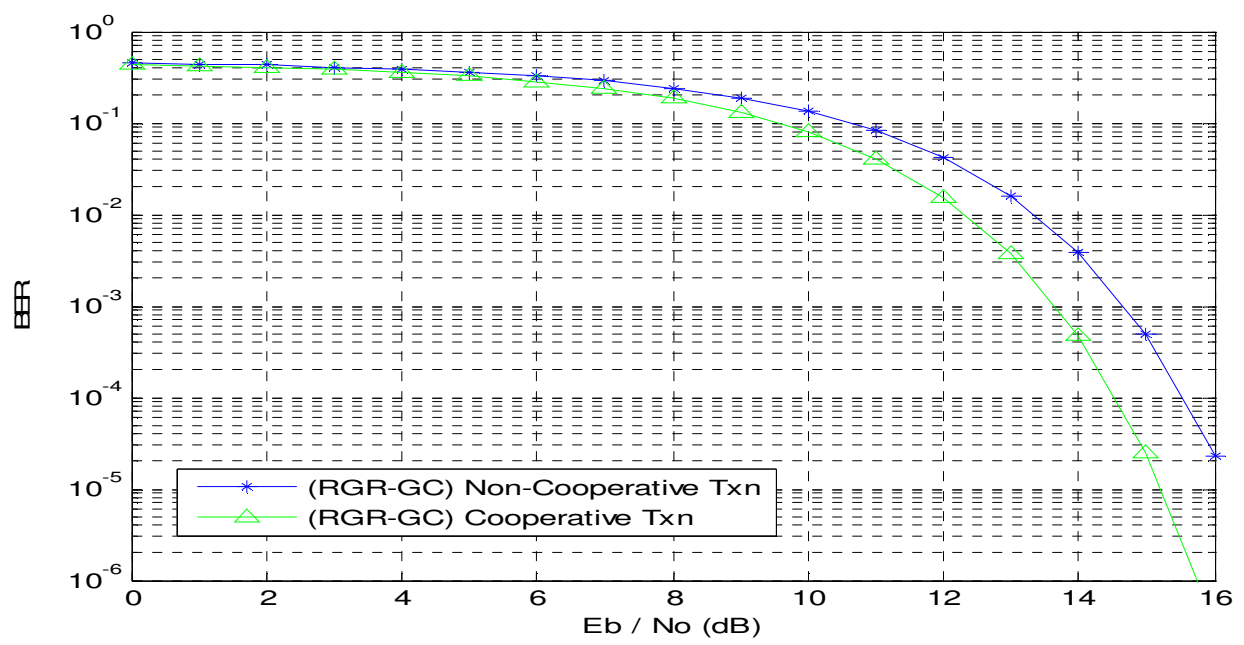

Figure. 5 BER performance analysis of the proposed RGR-GC inCooperative relaying scenario

From figure. 5, the BER performance analysis graph for the proposed RGR-GC in cooperative relaying transmission and non-cooperative transmission were plotted. It is inferred that, for a fixed BER of $10^{-3}$ the cooperative transmission method requires $13.5 \mathrm{~dB}$ (approx) and noncooperative transmission scheme requires $14.5 \mathrm{~dB}$ (approx). The BER performance of the proposed RGR_GC in cooperative transmission has better performance compared to the RGR_GC in Non-cooperative transmission. 


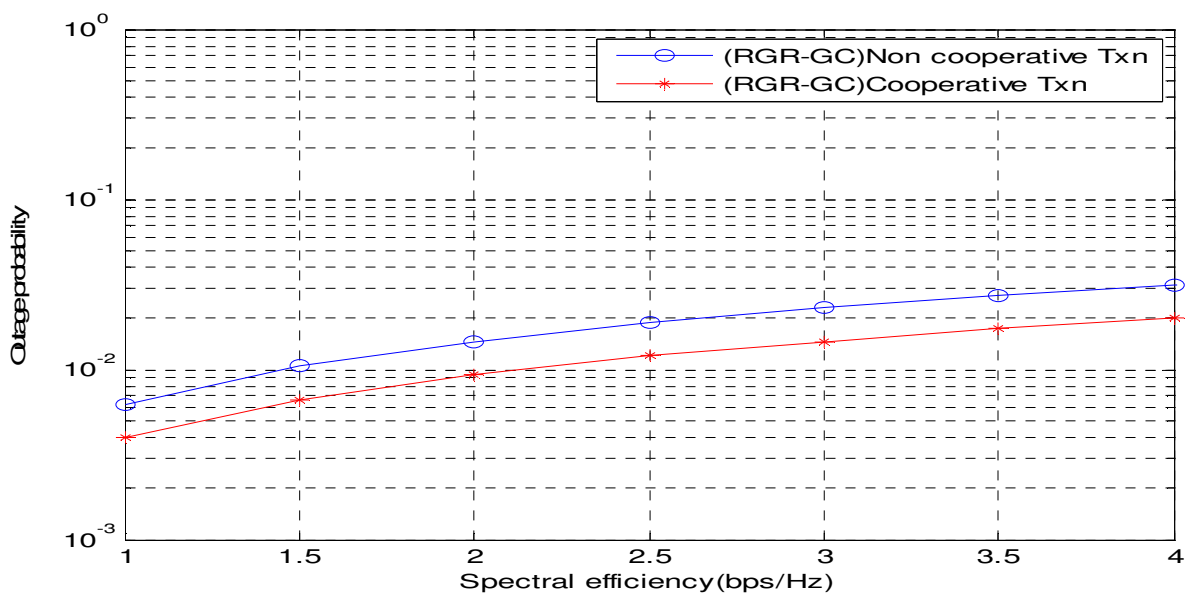

Figure 6. Outage Probability Versus Spectral Efficiency of (RGR-GC) in Cooperative relaying scenario

From figure 6, the performance graphs for RGR_GC in cooperative relaying method were plotted between outage probability and spectral efficiency. It is inferred that for a fixed spectral efficiency of $2 \mathrm{bps} / \mathrm{Hz}$ the proposed (RGR_GC) in cooperative transmission method has the outage probability of 0.005 (approx) and in non-cooperative transmission method has the outage probability of 0.01 (approx). Then, for fixed outage probability of $10^{-2}$, the spectral efficiency for cooperative transmission method and non-cooperative transmission is $2 \mathrm{bps} / \mathrm{Hz}$ and $1.5 \mathrm{bps} / \mathrm{Hz}$ respectively. The proposed RGR_GC in cooperative transmission has minimum outage probability and increased spectral efficiency compared to the RGR_GC in Non-cooperative transmission method. According to the system capacity condition, as the spectral efficiency increases the outage probability also gets increased.

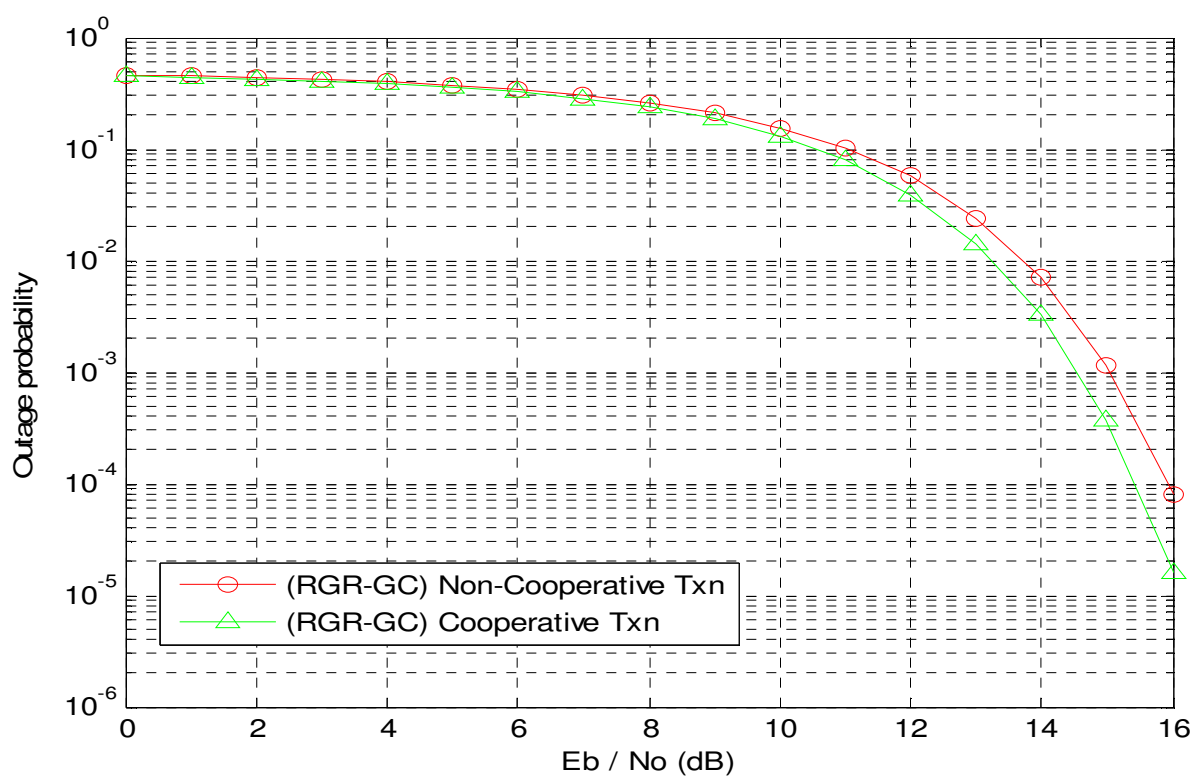

Figure 7. Outage Probability Versus Eb / No of (RGR-GC) in Cooperative relaying scenario

The BER performance analysis graph for the proposed RGR-GC in cooperative relaying transmission and non-cooperative transmission were plotted. From figure 7, it is inferred that, for a fixed $\mathrm{Eb} / \mathrm{No}$ of $15 \mathrm{~dB}$ the (RGR_GC) in cooperative transmission method has the outage probability of $1 \times 10^{-3}$ (approx) and in non-cooperative transmission method has the outage 
probability of $1.42 \times 10^{-4}$ (approx). The proposed RGR_GC in cooperative transmission has minimum outage probability compared to the RGR_GC in Non-cooperative transmission method.

\section{CONCLuSion}

In this paper, a modified golden code with the low complexity sphere decoder which uses tree search algorithm implemented in the nodes of the cooperative relaying system is proposed. The proposed decoder complexity is analyzed through simulation and it proves to exhibit less complexity compared to the conventional ML decoder. The single relay cooperative communication with DF protocol is considered. Simulation analysis is done and the simulation results shows that the proposed scheme achieves better BER performance, minimum outage probability and increased spectral efficiency, which is the need of the hour for the cooperative relaying scenario of wireless communication.

\section{REFERENCES}

[1] Z. Li, X.-G. Xia, and M. H. Lee, "A simple orthogonal space-time coding scheme for synchronous cooperative systems for frequency selective fading channels," IEEE Trans. Commun., vol. 58, no. 8, pp.2219-2224, Aug. 2010.

[2] Manav R. Bhatnagar , "Decode-and-Forward-Based Differential Modulation for Cooperative Communication System With Unitary and Nonunitary Constellations" , IEEE Transactions On Vehicular Technology, Vol. 61, No. 1, January 2012.

[3] Wang and Xia, "Asynchronous cooperative communication systems: A survey on signal designs", Science China Information Sciences, August 2011.

[4] Hongjiang Xiao, Qionghai Dai, Xiangyang Ji, and Wenwu Zhu, "A Novel JSCC Framework With Diversity-Multiplexing-Coding Gain Tradeoff for Scalable Video Transmission Over Cooperative MIMO, IEEE Transactions On Circuits And Systems For Video Technology, Vol. 20, No. 7, July 2010.

[5] Ehsan Yazdian,Mohammad Reza Pakravan, Adaptive Modulation Technique for Amplify and Forward Cooperative Diversity and Fairness Analysis, IEEE International Conference on Telecommunications, ICT June 2008.

[6] Michael Knox, Elza Erkip, Kshitij Kumar Singh, "Cooperative coding implementation at the physical layer", IEEE International Conference Acoustics Speech and Signal Processing (ICASSP), March 2010.

[7] Jinhong Yuan and Zhuo chen, "Distributed space time trellis codes for a cooperative system", IEEE Trans. on Wireless Communications, vol. 8, No. 11, October 2009.

[8] Manav R.Bhatnagar,and Are Hjorunges, "Amplify based double differential modulation for Cooperative communications", Proceedings in IEEE International conference on COMmunication Systems And NETworks, COMSNETS'09

[9] Soon Xin Ng, Kai Zhu and Lajos Hanzo, "Distributed source coding, channel coding and modulation for cooperative communications", VTC2010-FALL Ottawa, Sep 2010.

[10] Roderick Jaehoon and Huaping liu, "Transmission pattern selection for relay communication with Distributed space time codes", IEEE International Conference on Communication Systems, ICCS November 2008.

[11] Sheng Yang and Jean-Claude Belfiore, Optimal Space-Time codes for the MIMO amplify and forward cooperative channel, IEEE Trans. Inform. Theory, vol. 53, no. 2, February 2007.

[12] R. Nikjah and N. C. Beaulieu, "Achievable rates and fairness in rateless coded decode- and-forward half- duplex and full-duplex opportunistic relaying," in Proc. IEEE ICC, May 2008.

[13] R. U. Nabar, H. Bolcskei, and F. W. Kneubuhler, "Fading relay channels:performance limits and space- time signal design, “ IEEE J. Sel. Areas Commun., vol. 22, no. 6, pp. 1099-1109, Aug.2004.

[14] Andrej Stefanov and Elza Erkip, "Cooperative space-time coding for wireless networks", IEEE Trans. on Communications, vol. 53, No. 11, November 2005.

[15] J.C. Belfiore, G. Rekaya, and E. Viterbo, "The Golden Code: A $2 \times 2$ full rate Space-Time Code with Non Vanishing Determinants," IEEE Trans. on Inf. Theory, vol. 51, no 4, April 2005. 
[16] Ingrid Daubechies, C. Sinan Güntürk and Yang Wang, “The Golden Ratio Encoder”, IEEE Transactions on Information Theory, Vol. 56, No. 10, October 2010.

[17] M. O. Sinnokrot and J. R. Barry, "Fast Maximum- Likelihood Decoding of the Golden Code," IEEE Trans.Wireless Commun., vol. 9, no. 1, pp. 26-31, Jan. 2010.

[18] G. R.B. Othman, L. Luzzi and J.-C. Belfiore, "Algebraic Reduction for the Golden Code," in Proc. IEEE ICC 2009, Dresden, Germany, Jun.2009, pp. 1-5.

[19] Mohanned O. Sinnokrot, "Space-Time Block Codes with Low Maximum-Likelihood decoding Complexity”, Ph.D.Thesis, Georgia Institute of Technology, December 2009.

[20] G.S. Rajan and B.S.Rajan, "Multi-group ML Decodable Collocated and Distributed Space Time Block Codes," IEEE Trans. on Inf. Theory, vol.56, no.7, pp. 3221-3247, July 2010.

[21] K.Thilagam and K.Jayanthi, "Modified Golden Codes for Improved Error Rates through Low Complex Sphere Decoder" accepted by WiMoA 2013, will be published in Springer Proceedings, May 2013.

\section{Authors}

K.Jayanthi received B.E degree from University of Madras in 1997 and M.Tech degree in Electronics and Communication Engineering and Ph.D from Pondicherry University in 1999 and 2007 respectively. She has 13years of experience in teaching / research. She is currently serving as Associate Professor in Department of Electronics and Communication Engineering, Pondicherry Engineering College, Puducherry. To her credit, she has around 15 journal papers and presented 18 papers in International conferences. Her other areas of

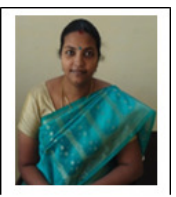
interest includes satellite communication, Wireless multimedia networks, Spread spectrum communication, Image processing, etc. She has authored a book on Qos Provisioning in Cellular Mobile Networks.

K.Thilagam received her B.Tech and M.Tech degrees in Electronics and Communication Engineering from Pondicherry Engineering College in 2004 and 2007 respectively. She has 5 years of experience in teaching / research. She is currently pursuing Ph.D in the area of Wireless Communication in Department of ECE from Pondicherry Engineering College, Puducherry. Her research activities are focussed on Cooperative Communication in Cellular Networks, mainly on Modulation and coding techniques.

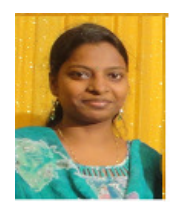

